\title{
Operative Wiederherstellung der Hornhautsensibilität - ein interdisziplinärer Fallbericht
}

\author{
Eva Györi · Christine Radtke · Gerald Schmidinger · Jan Lammer · Ursula Schmidt-Erfurth · Roman Dunavölgyi
}

Eingegangen: 10. Oktober 2018 / Angenommen: 22. März 2019 / Online publiziert: 11. April 2019

(c) Der/die Autor(en) 2019

\section{Zusammenfassung}

Hintergrund Läsionen des N. ophthalmicus führen $\mathrm{zu}$ neurotropher Keratopathie. Die bisherige Therapie war rein symptomatisch und führte nicht zur Verbesserung der Hornhautsensibilität. Eine rezent publizierte Operationsmethode ermöglicht eine Reinnervation der asensiblen Hornhaut durch Nervenfasern des kontralateralen N. supratrochlearis oder N. supraorbitalis über ein autologes Nerventransplantat. Wir beschreiben den ersten Fallbericht eines österreichischen Patienten mit neurotropher Keratopathie, dessen Hornhaut im Rahmen einer interdisziplinären Kooperation erfolgreich reinnerviert wurde.

Methodik Es wird der Fallbericht eines 59-jährigen Patienten mit vollständiger Asensibilität der linken Hornhaut nach Herpeskeratitis beschrieben. Bei dem Patienten wurde die Hornhaut durch Spendernerven des rechten N. supratrochlearis reinnerviert. Es wurde ein autologes Nerventransplantat eingebracht, welches End-zu-Seit an den N. supratrochlearis koaptiert wurde. Auf der Empfängerseite wurden die einzelnen Faszikel subkonjunktival, perilimbal angenäht. Der Patient wurde mit einer temporären Tarsorrhaphie versorgt.

Ergebnisse Sechs Monate postoperativ zeigte sich bei der Cochet-Bonnet-Ästhesiometrie ein Anstieg der Sensibilität im temporalen Bereich der Hornhaut,

\footnotetext{
E. Györi · C. Radtke

Klinische Abteilung für Plastische und Rekonstruktive Chirurgie, Universitätsklinik für Chirurgie, Medizinische Universität Wien, Wien, Österreich

G. Schmidinger · J. Lammer $\cdot$ U. Schmidt-Erfurth . Assoc. Prof. PD Dr. med. univ. R. Dunavölgyi $(\bowtie)$ Universitätsklinik für Augenheilkunde und Optometrie, Medizinische Universität Wien, Währinger Gürtel 18-20, 1090 Wien, Österreich

roman.dunavoelgyi@meduniwien.ac.at
}

sodass nun eine limbale Stammzelltransplantation sowie eine Keratoplastik geplant werden können.

Schlussfolgerung Die Wiederherstellung der Hornhautinnervation ermöglicht eine kausale Therapie der neurotrophen Keratopathie. In dieser interdisziplinären Kasuistik wird der erste österreichische Fall beschrieben.

Schlüsselwörter Neurotrophe Keratopathie · Hornhautulkus · Asensible Hornhaut - Reinnervation · Nerventransplantat

\section{Surgical restoration of corneal sensation - an interdisciplinary case report}

\section{Summary \\ Background Lesions of the ophthalmic branch of the trigeminal nerve lead to neurotrophic keratopathy. So far, therapeutic options were limited to symptomatic treatment, which did not improve corneal sensation. Recently, a novel operative technique was published where the insensate cornea was reinnervated by donor nerve fibers from the contralateral supratrochlear or supraorbital nerve using an autologous nerve graft. This case report describes the first Austrian patient with neurotrophic keratopathy who successfully un- derwent corneal reinnervation surgery in an interdis- ciplinary cooperation. \\ Methods A 59-year-old male patient presented with longstanding complete corneal insensitivity after her- pes keratitis. In an interdisciplinary cooperation the left insensate cornea was reinnervated with donor ax- ons from the contralateral supratrochlear nerve using an autologous sural nerve graft. On the donor side, an end-to-side neurorrhaphy was performed to coapt the nerve graft to the donor nerve branch. Distally, the sural nerve fascicles were separated, tunneled under the conjunctiva and coapted to the limbus. A tempo-}



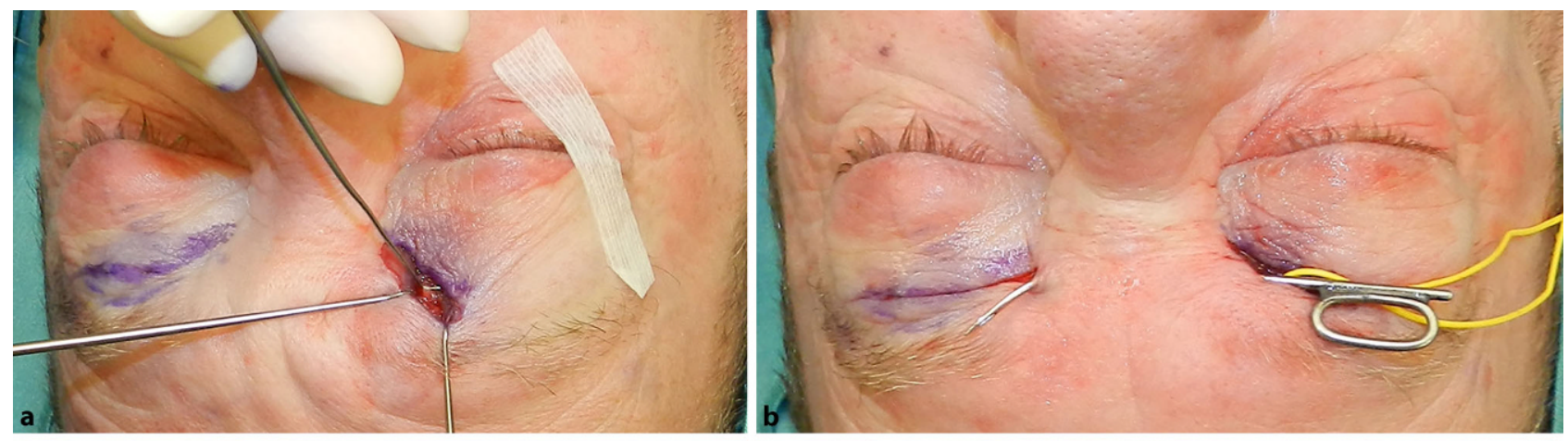

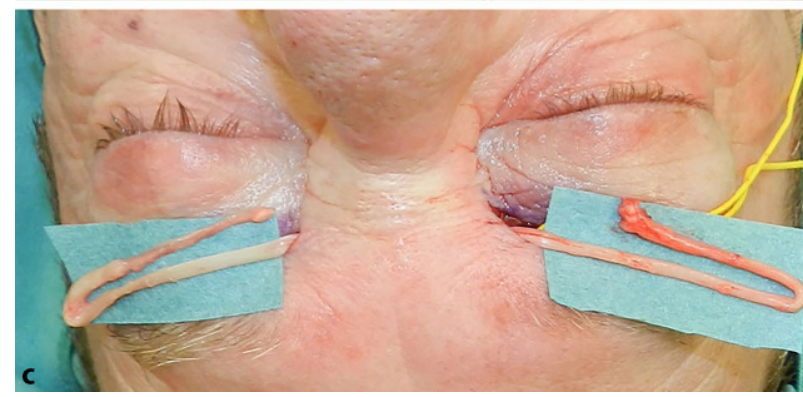

Abb. 1 Korneale Reinnervation mittels autologem Nerventransplantat. a Präparation des Spendernerven (kontralateraler N. supratrochlearis). b Subkutane Tunnelierung an der Ra-

rary tarsorrhaphy was performed to improve wound healing.

Results At 6 months postoperatively, Cochet-Bonnet esthesiometry showed improvement of corneal sensation in the temporal area of the cornea. The patient will now be prepared for limbal stem cell transplantation and keratoplasty.

Conclusion This interdisciplinary case report describes the first Austrian case of corneal reinnervation. This operative technique provides a causal therapeutic option for patients suffering from neurotrophic keratopathy.

Keywords Neurotrophic keratopathy - Corneal ulcer · Insensate cornea · Reinnervation · Nerve graft

Die sensible Innervation der Hornhaut erfolgt durch den Nervus (N.) ophthalmicus des N. trigeminus [1]. Der vollständige Verlust der Hornhautsensibilität führt zu schweren Augenoberflächenstörungen bis hin zu (neurotrophen) Hornhautulzera. Im weiteren Verlauf kann es in fortgeschrittenen Fällen zur Einschmelzung der Hornhaut kommen [2]. Darüber hinaus führt die intensive Therapie mit befeuchtenden Augentropfen zu einer Einschränkung der Lebensqualität der Patienten.

Neben angeborenen gibt es zahlreiche erworbene Ursachen einer Asensibilität der Hornhaut. Die häufigsten erworbenen Ätiologien sind virale Infektionen (Herpes simplex und Herpes zoster). Des Weiteren führen Verätzungen der Hornhaut, Traumata, intrakranielle Pathologien und systemische Erkrankungen

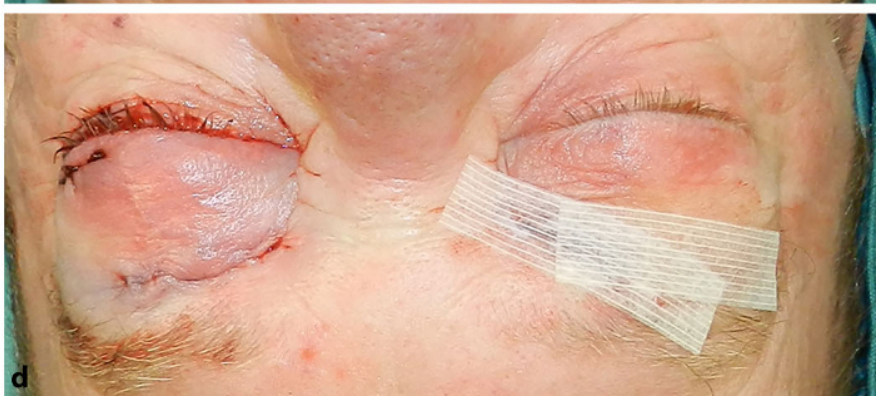

dix nasii. c Autologes Nerventransplantat (N. suralis) wird eingebracht. d Wundverschluss und temporäre Tarsorrhaphie

(z.B. Diabetes mellitus, demyelinisierende Erkrankungen) zum Verlust der Hornhautsensibilität [3-5]. Angeborene korneale Asensibilität tritt meist bilateral auf und kann mit weiteren angeborenen Ausfällen des N. trigeminus verbunden sein [1]. Angeborene Fälle können isoliert, oder in Zusammenhang mit syndromalen Fehlbildungen wie dem Goldenhar-Syndrom, VACTERL-Assoziation oder Moebius-Syndrom auftreten [1]. Pathophysiologisch kommt es zu einer Veränderung der Neurotransmitterkonzentration in den sensorischen und sympathischen Nervenfasern. Experimentelle Studien konnten auch den Einfluss von veränderten Spiegeln von cAMP, cGMP und Substanz $\mathrm{P}$ auf die Regeneration auf Hornhautepithelzellen darstellen $[4,6]$.

Konservative Therapieoptionen umfassen Tränenersatzmittel, epithelisationsfördernde Augensalben, Verbandskontaktlinsen, Uhrglasverbände, sowie im Falle von Infektionen antibiotische, antivirale oder antifungale Therapieoptionen [1].

Terzis et al. publizierten die Erstbeschreibung einer Operationstechnik bei der über einen bikoronaren Zugang ein direkter Nerventransfer des N. supratrochlearis und N. supraorbitalis der gesunden Seite die asensible Kornea in unilateralen Fällen reinnervierte [2]. Obwohl die Hornhautsensibilität erfolgreich wiederhergestellt werden konnte, wurde diese Methode aufgrund der Invasivität nicht weiter aufgegriffen.

In den letzten Jahren beschrieb eine Arbeitsgruppe der University of Toronto eine minimalinvasive Operationstechnik, bei der Nervenfasern des N. supratrochlearis über ein autologes Nerventransplantat vom N. suralis zur asensiblen Hornhaut geleitet wer- 

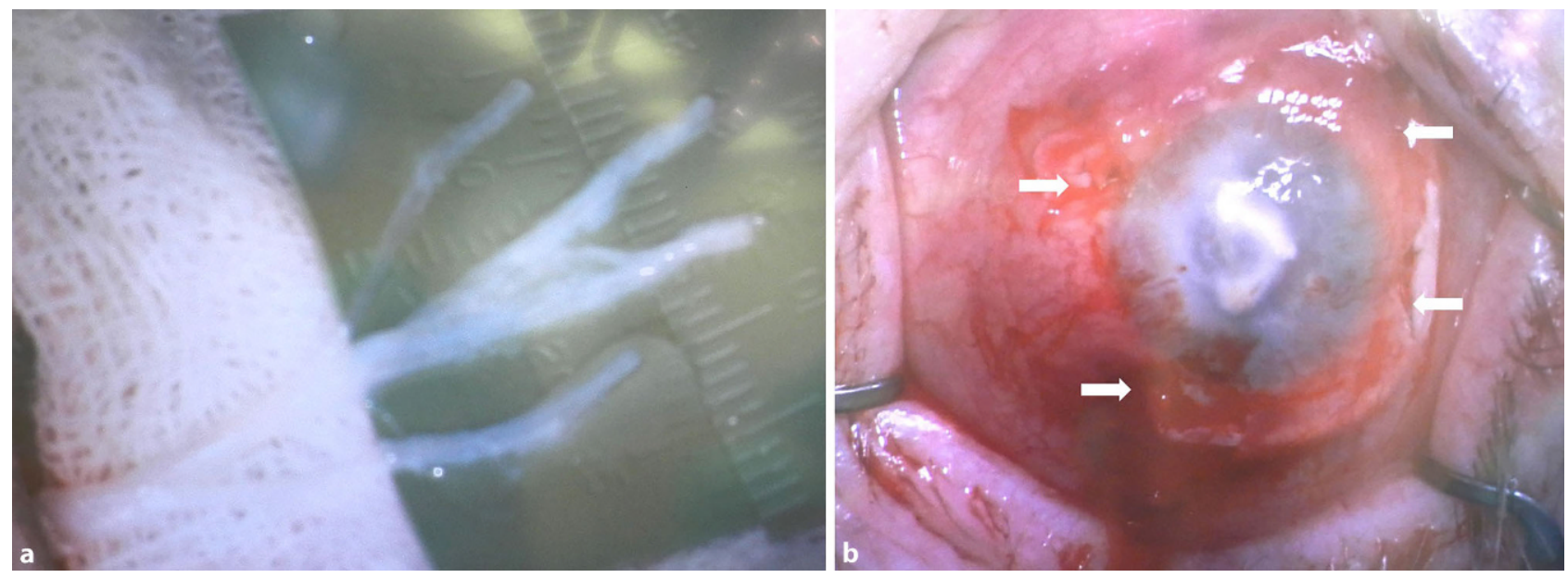

Abb. 2 Mikrochirurgische Präparation und Nervennaht. a Auftrennung der Faszikel des N.-suralis-Transplantats. b Perilimbale Nervennaht der einzelnen Faszikel (Pfeile)

den [7, 8]. Die Ergebnisse der ersten 5 Patienten wurden publiziert und zeigten eine beginnende Verbesserung der Sensibilität der Hornhaut 6 Monate nach der Operation [8]. In einer konfokalen Hornhautmikroskopie konnten Fung et al. die Reinnervation der Hornhaut bei einer Fallserie von 6 Patienten in vivo nachweisen [9].

An der Medizinischen Universität Wien konnte im Rahmen einer interdisziplinären Kooperation zwischen der Universitätsklinik für Augenheilkunde und Optometrie und klinischen Abteilung für Plastische und Rekonstruktive Chirurgie bei einem ersten Patienten mit vollständiger Asensibilität der Hornhaut die Innervation der Kornea verbessert werden. Wir beschreiben im Folgenden die Operationstechnik sowie den Krankheits- und Heilungsverlauf dieses Patienten. Nach unserem Wissensstand ist dies die erste operative Behandlung der kornealen Asensibilität in Österreich.

\section{Kasuistik}

Wir berichten über den Fall eines 59-jährigen Patienten, welcher im September 2016 in der Hornhautambulanz der Universitätsklinik für Augenheilkunde und Optometrie der Medizinischen Universität Wien vorstellig wurde. Anamnestisch besteht bei dem Patienten eine dauerhafte und vollständige Asensibilität der Hornhaut links nach einer abgelaufenen Herpeskeratitis. Der Patient war wenige Monate vor der Erstvorstellung in unserer Ambulanz aufgrund einer schwerwiegenden Keratitis stationär an einer Augenabteilung eines Wiener Spitals aufgenommen worden. Bei der Erstvorstellung in der Hornhautambulanz wurden ein großflächiges Hornhautulkus mit Gewebedefekt, eine vollständig fehlende Hornhautsensibilität sowie Hornhautnarben durch vorangegangene Keratitiden festgestellt. Der Patient wurde mittels therapeutischer Kontaktlinse, topischer antibiotischer, antiinflammatorischer und benetzender Therapie behandelt. Augenab- striche wurden zur Feststellung eines infektiösen Erregers abgenommen. Eine operative Sanierung mittels penetrierender Keratoplastik (PKP) wurde aufgrund des St.p. Herpeskeratitis mit vollständiger Asensibilität prognostisch als ungünstig eingeschätzt. Im Laufe der folgenden Monate schloss sich das Epithel über dem verdünnten Ulkusrand, die Erosio corneae blieb zentral jedoch erhalten. Es setzte auch eine zunehmende Trübung der Hornhaut zentral ein. Zu diesem Zeitpunkt wurde eine Reinnervationsoperation in Erwägung gezogen, es erschien jedoch notwendig, die Abheilung der Erosio corneae abzuwarten. Im März 2017 wurde bei einem erneuten Augenabstrich eine Infektion mit Candida parapsilosis entdeckt, welche in den Wochen darauf mit Fluconazol-Augentropfen erfolgreich behandelt werden konnte.

Bei geschlossenem Hornhautepithel wurde nun im Frühjahr 2017 vor Erwägung einer PKP die Reinnervationsoperation der linken Hornhaut geplant. Präoperativ zeigte sich unverändert keinerlei Hornhautsensibilität links mit der Testung mittels Cochet-Bonnet-Ästhesiometer (Luneau Technology, Leeds, West Yorkshire, UK). Ebenso zeigte sich eine Pannusbildung bei kompletter $\left(360^{\circ}\right)$ limbaler Stammzellinsuffizienz.

Anfang Juni 2017 wurde die operative korneale Reinnervation mit temporaler Tarsorrhaphie in Intubationsnarkose durchgeführt (Abb. 1 und 2). Die Operation war Teil einer laufenden klinischen Studie, welche von der Ethikkommission der Medizinischen Universität Wien bewilligt wurde (Protokoll Nummer 1826/2016). Zunächst wurde das N.-suralisTransplantat im Bereich des rechten Unterschenkels minimalinvasiv entnommen. Anschließend wurde der N. supratrochlearis auf der gesunden, rechten Seite über eine $2 \mathrm{~cm}$ große kurze transverse Hautinzision im Bereich des medialen Oberlides rechts dargestellt. Nach symmetrischer Hautinzision am linken Oberlid wurde ein Tunnel über die Radix nasii zur subkutanen Platzierung des Nerventransplantates präpariert. Das Nerventransplantat wurde auf der linken Seite 
durch die Hautinzision geborgen. Nun wurde mit einer Fasziennadel von der Hautinzision am medialen Teil des linken Oberlides ein Tunnel zum superomedialen Fornix des linken Auges präpariert. Das Suralistransplantat wurde folgend mit der Fasziennadel durch den Fornix geführt und dort geborgen (Abb. 1). Die einzelnen Faszikel des N. suralis wurden nach Abpräparation des Epineuriums getrennt und durch subkonjunktivale Tunnel in allen 4 Quadranten in Richtung des Limbus corneae in $2 \mathrm{~mm}$ Abstand von ebendiesem mit 10-0-Nylonnähten an der Sklera fixiert (Abb. 2). Nach Bindehautverschluss wurde daraufhin das Nerventransplantat gekürzt, wobei hier auf eine spannungsfreie Motilität des linken Auges geachtet wurde. Das Nerventransplantat wurde nun proximal mit 10-0-Nylonnähten End-zu-Seit an den gesunden rechten N. supratrochlearis koaptiert, wodurch die Kontinuität des Spendernervs sowie dessen Funktion erhalten blieben und es zu keinen sensiblen Ausfällen kam. Nach den Hautnähten wurde zum Schutz der Hornhaut und des Nerventransplantats eine temporale Tarsorrhaphie durchgeführt. Die Hautnähte wurden mit Steristrips versorgt. Am linken Auge wurde ein Augenverband mit Gentamicin (Gentax)-Augensalbe angelegt.

Der Patient konnte am dritten postoperativen Tag in die häusliche Pflege entlassen werden. Die temporale Tarsorrhaphie wurde 2 Wochen später eröffnet. Der postoperative Heilungsverlauf war komplikationsfrei. Es kam in den folgenden Monaten zu keinen neuen Infektionen der Hornhaut oder Epitheldefekten. Die Nerventransplantate heilten subkonjunktival entzündungsfrei $\mathrm{ab}$.

Bei der Kontrolle 6 Monate nach Eröffnung der Tarsorrhaphie zeigte sich bei der Cochet-Bonnet-Ästhesiometrie ein Anstieg der Sensibilität im temporalen Bereich der Hornhaut. Aufgrund der verbesserten Hornhautsensibilität werden bei dem Patienten nun am linken Auge eine limbale Stammzelltransplantation sowie später eine PKP geplant.

\section{Schlussfolgerungen}

Wir beschreiben den österreichweit ersten Fall einer operativen Wiederherstellung der Hornhautsensibilität bei einer schwerwiegenden neurotrophen Keratopathie. Durch die erzielte Reinnervation der Hornhaut ist nun im Folgenden die Prognose für weitere opera- tive Maßnahmen wie Stammzelltransplantation oder Keratoplastik deutlich verbessert.

Funding Open access funding provided by Medical University of Vienna.

\section{Einhaltung ethischer Richtlinien}

Interessenkonflikt E. Györi, C. Radtke, G. Schmidinger, J. Lammer, U. Schmidt-Erfurth und R. Dunavölgyi geben an, dass kein Interessenkonflikt besteht.

Ethische Standards Der Patient gab seine Einwilligung zur Teilnahme an der Studie und unterzeichnete die Patienteninformation.

Open Access Dieser Artikel wird unter der Creative Commons Namensnennung 4.0 International Lizenz (http:// creativecommons.org/licenses/by/4.0/deed.de) veröffentlicht, welche die Nutzung, Vervielfältigung, Bearbeitung, Verbreitung und Wiedergabe in jeglichem Medium und Format erlaubt, sofern Sie den/die ursprünglichen Autor(en) und die Quelle ordnungsgemäß nennen, einen Link zur Creative Commons Lizenz beifügen und angeben, ob Änderungen vorgenommen wurden.

\section{Literatur}

1. Bonini S, et al. Neurotrophic keratitis. Eye (Lond). 2003;17(8):989-95.

2. Terzis JK, Dryer MM, Bodner BI. Corneal neurotization: anovel solution to neurotrophickeratopathy. Plast Reconstr Surg. 2009;123(1):112-20.

3. Mathen MM, etal. Congenital corneal anesthesia: a series of four case reports. Cornea. 2001;20(2):194-6.

4. Ramaesh K, et al. Congenital corneal anesthesia. Surv Ophthalmol. 2007;52(1):50-60.

5. Rosenberg ML. Congenital trigeminal anaesthesia. Areview and classification. Brain. 1984;107(Pt4):1073-82.

6. Cavanagh HD, Colley AM. The molecular basis of neurotrophic keratitis. Acta Ophthalmol Suppl. 1989;192:115-34.

7. Bains RD, et al. Corneal neurotization from the supratrochlear nerve with sural nerve grafts: a minimally invasive approach. Plast Reconstr Surg. 2015;135(2):397e-400e.

8. ElbazU, etal. Restoration of corneal sensation with regional nerve transfers and nerve grafts: a new approach to a difficult problem. JAMA Ophthalmol. 2014;132(11):1289-95.

9. FungSSM, et al. In vivo confocal microscopy reveals corneal reinnervation after treatment of neurotrophic keratopathy with corneal neurotization. Cornea. 2018;37(1):109-12.

Hinweis des Verlags Der Verlag bleibt in Hinblick auf geografische Zuordnungen und Gebietsbezeichnungen in veröffentlichten Karten und Institutsadressen neutral. 